\title{
Elaboración de chocolate de siete genotipos experimentales de cacao (Theobroma cacao L.) seleccionados en la Finca Experimental La Represa
}

\section{Elaboration of chocolate of seven experimental genotypes of cocoa (Theobroma cacao L.) selected in the Experimental Farm La Represa}

\author{
Tigselema Zambrano Solanyi Marley ${ }^{\circ}{ }^{\bullet}$ Vera Chang Jaime Fabián ${ }^{1}$, Ordoñez Choez Shirley Estefanía², \\ Segovia Freire Gerardo Francisco ${ }^{1}$, Vásconez Montúfar Gregorio Humberto ${ }^{1}$, Rosero Zambrano Joel Eduardo ${ }^{1}$ \\ ${ }^{1}$ Universidad Técnica Estatal de Quevedo, Facultad de Ciencias Pecuarias, Finca Experimental "La María” $\mathrm{km} 7$ \\ de la vía Quevedo-El Empalme. Recinto San Felipe, cantón Mocache, provincia de Los Ríos, Ecuador. \\ Solanyi.tigselema2013@uteq.edu.ec,•jverac@uteq.edu.ec,gsegovia@uteq.edu.ec, \\ gvasconez@uteq.edu.ec,jo_ed@outlook.com \\ ${ }^{2}$ Grupo Manobanda S.A., km 1 de la vía Valencia-Quevedo, cantón Quevedo, provincia de Los Ríos, \\ shirleyecho.ordonez@uteq.edu.ec
}

Rec.: 31.10.2018. Acept.: 13.12.2018 Publicado el 30 de diciembre de 2018

\begin{abstract}
Resumen
El objetivo de esta investigación es evaluar las propiedades químicas y cualidades organolépticas de chocolate de siete genotipos experimentales de cacao (Theobroma cacao L.) seleccionados en la finca experimental "La Represa", de la Universidad Técnica Estatal de Quevedo. Se utilizó un diseño completamente al azar (DCA) con siete tratamientos y tres repeticiones y las medias fueron comparadas a través de la prueba de Tukey $(\mathrm{p} \leq 0.05)$. Se determinaron las propiedades químicas (Grasa, acidez y energía) y las cualidades organolépticas (generalidades, sabores básicos, sabores específicos, defectos) empleando una prueba descriptiva al chocolate negro degustado por un panel de 10 jueces semi entrenados. Se realizó un análisis económico el cual varió entre tratamientos debido a las formulaciones utilizadas. Para la variable grasa el mejor tratamiento fue el DICYT-H 258 (37.83), la acidez con menor valor lo obtuvo el CCN51 (2.04) estadísticamente diferente del DICYT-H 259 (8.79), para energía no hubo diferencias significativas, se obtuvo una media general de $607 \mathrm{Kcal}$. Para las variables organolépticas el DICYT-H 262 obtuvo un perfil floral (Sabor Arriba). El mayor costo fue para el tratamiento CCN-51 con USD 51.20, el menor costo fue el DICYT-H 258 con USD 40.20 por el uso de más aditivos que los otros tratamientos.
\end{abstract}

Palabras clave: cacao, genotipos experimentales, calidad química y organoléptica.
The objective of this research is to evaluate the chemical and organoleptic properties of chocolate from seven interclonal cocoa crosses (Theobroma cacao L.) selected in the Experimental Farm "La Represa", of the State Technical University of Quevedo. A completely randomized design (DCA) was used with seven treatments and three repetitions and the means were compared through the Tukey test $(\mathrm{p} \leq 0.05)$. he chemical properties (fat, acidity and energy) and the organoleptic qualities (generalities, basic flavors, specific flavors, defects) were determined using a descriptive test of dark chocolate tasted by a panel of 10 semi-trained judges. An economic analysis was carried out which varied between treatments due to the formulations used. For the fat variable the best treatment was the DICYT-H 258 (37.83), the acidity with lower value was obtained by the CCN-51 (2.04) statistically different from the DICYT-H 259 (8.79), for energy there were no significant statistical differences, a general average of $607 \mathrm{Kcal}$ was obtained. For the organoleptic variables, the DICYT-H 262 obtained a floral profile (Flavor Above). The highest cost was for the CCN-51 treatment with USD 51.20, the lowest cost was the DICYT-H 258 with USD 40.20 since more additives were used than in the other treatments.

Keywords: cocoa, interclonal crosses, chemical and organoleptic quality. 


\section{Introducción}

$\mathrm{E}$ cacao (Theobroma cacao L.) es una fruta de origen tropical, su árbol tiene flores pequeñas y pétalos largos, su fruto es leñoso de forma alargada, aparece en la copa de los árboles y debajo de sus ramas. Dependiendo del tipo de cacao pueden ser de color amarillo, blanco, verde o rojo (Molina, 2017). Es un árbol procedente de América, sus granos se pueden utilizar como ingrediente para alimentos, su uso se remonta a la época de los mayas, aztecas e incas, y desde entonces se ha usado tanto para fines nutricionales como médicos (Fernández, 2016).

La producción de cacao se realiza principalmente en la costa y amazonía del Ecuador, las provincias de mayor producción son Los Ríos, Guayas, Manabí y Sucumbíos (Proecuador, 2017). Tradicionalmente se ha sostenido que el punto de origen de la domesticación del cacao se encontraba en Mesoamérica entre México, Guatemala y Honduras, donde su uso está atestiguado alrededor de 2000 años antes de Cristo. No obstante, estudios recientes demuestran que por lo menos una variedad de cacao tiene su punto de origen en la Alta Amazonía y que ha sido utilizada en la región por más de 5000 años (Ledesma, 2017).

El cacao tiene características distintivas de aroma y sabor buscadas por los fabricantes de chocolate. Ecuador, por sus condiciones geográficas y su riqueza en recursos biológicos, es el productor por excelencia de cacao fino de aroma (63\% de la producción mundial) proveniente de la variedad Nacional cuyo sabor ha sido reconocido durante siglos en el mercado internacional. Este tipo de grano es utilizado en todos los chocolates refinados (Maldonado, 2016).

En Latinoamérica frecuentemente los productores de cacao han sido obligados a abandonar sus plantaciones debido a los bajos rendimientos producidos por plagas, enfermedades o incorporación limitada de buenas prácticas agrícolas. Actualmente, países como Bolivia, Colombia, Costa Rica, Ecuador, Perú y México producen aproximadamente el $80 \%$ del cacao mundial, representando 1.7 millones de hectáreas. Sin embargo, el promedio de productividad permanece bajo (Foundation, 2014), debido a esta situación, instituciones de varios países desarrollan Programas de Mejoramiento Genético de Cacao.

La cosecha y pos cosecha del cacao involucra un proceso de recolección manual con machetes, fermentación, secado y clasificación, es decir, se prepara la materia prima para la elaboración del chocolate. Los procesos de fermentación y tostado generan diferencias sutiles en el color y sabor del chocolate resultante. Para la elaboración del chocolate se necesita una mezcla de cacao, o polvo de cacao, manteca de cacao y azúcar.
Después, según el producto que se desee obtener, se añaden otros ingredientes como leche, almendras, avellanas, frutas, y otros. Las fases de la fabricación del chocolate son: tostado, molienda, mezcla, molido fino, conchado, templado, moldeado, y envasado (Oliveras, 2007).

El consumo de chocolate tiene algunos beneficios para la salud humana por el contenido de antioxidantes, modula la función plaquetaria e inflamación y disminuye la presión arterial sistólica y diastólica. Aunque no existe consenso sobre la cantidad óptima a consumir, puede sugerirse que el consumo diario de chocolate rico en cacao (y polifenoles) es una buena elección para reducir, al menos parcialmente, el riesgo cardiovascular (Gómez et al., 2011).

Dependiendo de la cantidad de polvo de cacao y manteca mezclados junto a azúcares refinados y leche surgen los distintos tipos de chocolate (Fernández, 2016). Existe una gran variedad de chocolates cuyas denominaciones están relacionadas de alguna manera con los productos que son mezclados con la pasta de cacao. Sin embargo, esta casi infinita variedad parte de tres o cuatro materias primas básicas, que deben su denominación a su contenido de cacao: Chocolate negro, Chocolate con leche, Chocolate blanco, Chocolate líquido (Oliveras, 2007).

\section{Materiales y métodos}

T a investigación se realizó entre los meses de marzo hasta octubre del año 2018. La recolección de mazorcas de genotipos experimentales de cacao se realizó en la Finca Experimental "La Represa" de la Universidad Técnica Estatal de Quevedo, localizada en el km 7.5 recinto "Faita" de la Vía Quevedo -San Carlos, provincia de Los Ríos. Su ubicación geográfica es $1^{\circ} 03^{\prime} 18^{\prime \prime}$ de latitud Sur y $79^{\circ} 25^{\prime} 24^{\prime \prime}$ de longitud Oeste, a una altura de 73 metros sobre el nivel del mar. En el laboratorio de chocolate de la empresa Grupo Manobanda ubicada en el $\mathrm{km} 1$ de la vía ValenciaQuevedo se efectuó el proceso de elaboración de barras de chocolate negro. Los análisis químicos se realizaron en el Laboratorio de bromatología en la Finca Experimental "La María", de la Universidad Técnica Estatal de Quevedo, ubicada en el km 7 de la Vía Quevedo-El Empalme. Recinto San Felipe, cantón Mocache, provincia de Los Ríos, situada entre las coordenadas geográficas de $01^{\circ} 06^{\prime}$ de latitud Sur y $79^{\circ}$ 29' de longitud Oeste, a una altitud de $120 \mathrm{msnm}$ con una temperatura media de $25.8^{\circ} \mathrm{C}$.

La fermentación de los cruces experimentales se realizó por 4 días, mientras, el Testigo CCN-51 tuvo una fermentación de 5 días. Se empleó un diseño completamente al azar, con siete tratamientos, seis 
genotipos experimentales pertenecientes al programa de cacao de la Dirección de Investigación Científica y Tecnológica de la UTEQ, y un testigo comercial de origen trinitario $(\mathrm{CCN}-51)$, con tres repeticiones. El análisis estadístico se realizó mediante el análisis de varianza ANDEVA y las medias fueron comparadas a través de la prueba Tukey $(\mathrm{p} \leq 0.05)$, con la utilización del software para análisis estadístico de versión libre. Se realizó análisis organoléptico a las barras de chocolate negro con un panel constituido por 10 jueces semi entrenados, en base a la valoración de criterios organolépticos como: sabores básicos (acidez, amargor, astringencia), específicos (floral, futal, nuez, dulzor) y defectos (moho, quemado, contaminado).

Los análisis químicos incluyeron las variables: energía, acidez y grasa. Para obtener la grasa, se colocaron las muestras en estufa a $110^{\circ} \mathrm{C}$ por 2 horas, luego fueron transferidos al desecador por 30 minutos, se pesó $1 \mathrm{~g}$ de muestra colocadas en el interior del dedal, tapadas con algodón y ubicadas en el porta dedal, llevando los ganchos metálicos en el equipo goldfish según la norma técnica (NTE INEN 174 y 535). Se empleó la siguiente fórmula:

Grasa $(\%)=(\mathrm{W} 2-\mathrm{W} 1) / \mathrm{W} 0 \times 100$

Dónde:

$\mathrm{W} 0=$ Peso de la muestra

$\mathrm{W} 1=$ Peso del vaso beaker vacío

$\mathrm{W} 2=$ Peso del vaso más la grasa

En el cálculo de la acidez, se tomaron $10 \mathrm{~g}$ de muestra y se colocó en un matraz volumétrico de $250 \mathrm{ml}$, después se añadió $50 \mathrm{ml}$ de agua destilada, se agitó vigorosamente, se adicionó 5 gotas de fenolftaleína al $1 \%$ como indicador, se agregó gota a gota la solución
$\mathrm{NaOH}$, se tituló hasta que aparezca el color rosa y este permanezca 15 segundos, finalmente se tomó la lectura en la bureta de la cantidad de $\mathrm{NaOH}$ usada para neutralizar la acidez de la muestra.

$\mathrm{Ac}(\%)=\mathrm{A} * \mathrm{~B} * \mathrm{C} D * 100$

Para el cálculo de la Energía, se utilizó la fórmula:

$H g=\frac{T w-e 1-e 2-e 3}{m}$

Donde:

$\mathrm{Hg}=$ Calor de combustión $\mathrm{Cal} / \mathrm{g}$

$\mathrm{T}=$ Temperatura final- Temperatura inicial

$\mathrm{W}=$ Energía equivalente del calorímetro 2410.16

e1= Mililitros consumidos de sol. Carbonato de sodio

e2 $=(13.7 * 1.02)$ peso de la pastilla

$\mathrm{e} 3=\mathrm{cm}$ de alambre restante $* 2.3$

$\mathrm{m}=$ Peso de la pastilla

El análisis económico se basó en costos variables y costos fijos, a partir de los cuales se calculó el costo total por tratamiento.

\section{Resultados y discusión}

\section{Porcentaje de grasa}

Se muestra las concentraciones del porcentaje grasa presente en las barras de chocolate, los valores mayores correspondieron a las muestras DICYT-H 258 (37.83) y el DICYT-H 259 (36.58); mientras, los menores valores se registraron en CCN-51 (22.86) y DICYT-H 262 (31.41). La media general de 33.34 \pm 0.72 (Figura 1), con un Coeficiente de variación de $15.31 \%$. Salinas et al. (2012), al estudiar el contenido de grasa y ácidos grasos $(\mathrm{AG})$ en chocolates de mayor consumo en Venezuela, identificaron y cuantificaron

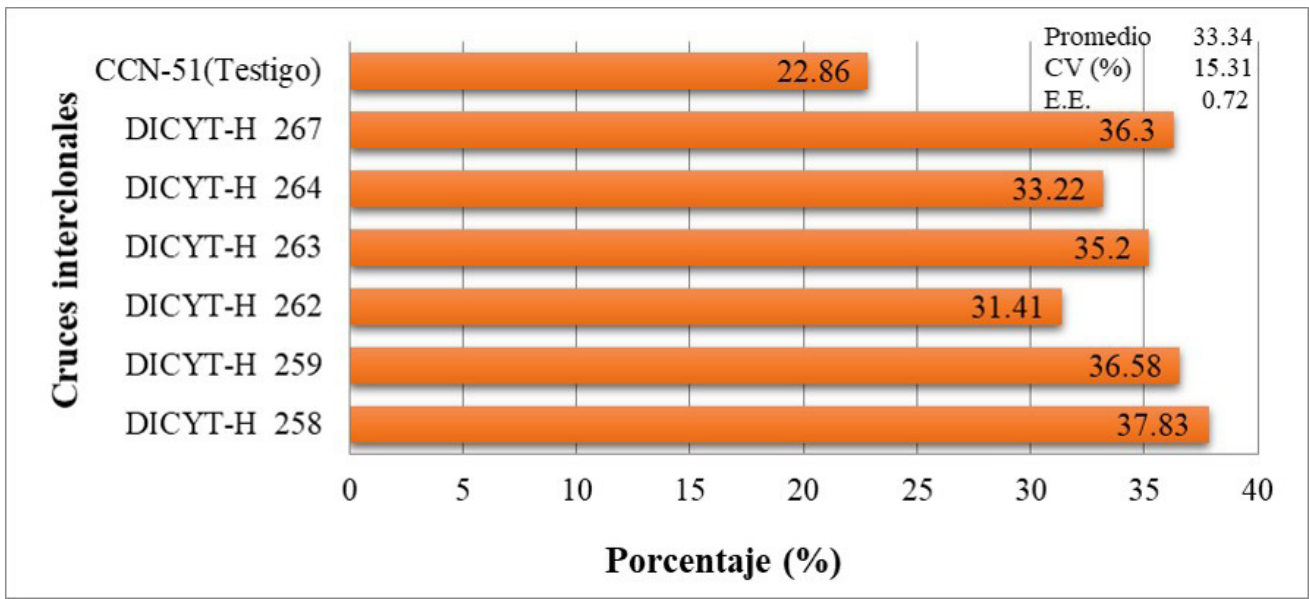

Figura 1. Resultados de los análisis de grasa realizados en las barras de chocolate 
los AG obteniendo alta variabilidad en el contenido de los saturados, monoinsaturados y poliinsaturados. Los valores mayores entre las muestras lo obtuvieron barra blanca (40.00) y barra de leche (37.0), mientras, los menores valores registrados fueron Chocolate tipo polvo (1.38) y análogos de chocolate Untable Tipo MC I (25.0).

Afoakwa et al. (2011), mencionan valores de grasa mayores a los obtenidos en las barras de chocolate negro, en granos sin fermentar (55.2\%) y el menor valor en granos fermentados a los 21 días (50.5\%).

\section{Acidez}

Se observa diferencias significativas de acidez presente en las barras de chocolate, los valores mayores entre las muestras lo obtuvieron DICYT-H 259 (8.79) y el DICYT-H 267 (5.89); mientras, los menores valores correspondieron a CCN-51 (2.04) y DICYT-H 258 (3.65), siendo la media general de $5.22 \pm 1.55$ y un Coeficiente de variación de $25.70 \%$ (Cuadro 1). Lares et al. (2016) caracterizaron las propiedades fisicoquímicas y el perfil de ácidos grasos de los granos de cacao de la cosecha denominada "San Juanera" en la región de Chuao y en diferentes etapas del beneficio, obteniendo valores de acidez menores a los obtenidos en las barras de chocolate negro, siendo el valor mayor en Granos fermentados (1.83) y el menor valor en Granos fermentados, secados al sol y tostados (1.30).

Homem et al. (2017), en la región cacaotera de Bahía, Brasil obtuvieron valores de acidez similares a los obtenidos en esta investigación (7.1 en granos fermentados). Al evaluar la influencia de las épocas de cosecha (invierno y verano) sobre las características

Cuadro 1. Valores registrados para las variables químicas: acidez y energía en barras de chocolate negro

\begin{tabular}{lcr}
\hline Tratamientos & $\begin{array}{c}\text { Acidez } \\
(\mathbf{\%})\end{array}$ & $\begin{array}{c}\text { Energía } \\
(\mathbf{K c a l} / \mathbf{1 0 0} \mathbf{g})\end{array}$ \\
\hline DICYT-H 259 & $8.79 \mathrm{a}$ & $634 \mathrm{a}$ \\
DICYT-H 267 & $5.89 \mathrm{a} \mathrm{b}$ & $628 \mathrm{a}$ \\
DICYT-H 264 & $5.67 \mathrm{a} \mathrm{b}$ & $558 \mathrm{a}$ \\
DICYT-H 262 & $5.63 \mathrm{a} \mathrm{b}$ & $619 \mathrm{a}$ \\
DICYT-H 263 & $4.89 \mathrm{a} \mathrm{b}$ & $627 \mathrm{a}$ \\
DICYT-H 258 & $3.65 \mathrm{a} \mathrm{b}$ & $616 \mathrm{a}$ \\
CCN-51 (Testigo). & $2.04 \mathrm{~b}$ & $567 \mathrm{a}$ \\
\hline Promedio & 5.22 & 607 \\
CV (\%) & 25.70 & 8.02 \\
\hline
\end{tabular}

Medias con una letra común no son significativamente diferentes según la prueba de Tukey $(\mathrm{p}>0.05)$ físicas, sensoriales y bromatológicas del cacao nacional perteneciente al jardín clonal de la ESPAM MFL, Ruíz et al. (2014), obtuvieron acidez para verano menores a los registrados en esta investigación, siendo mayor en el Clon EET-48 (0.98) y menor en EET-103 (0.52). Guzmán et al. (2014), indican nieveles de acidez entre 0.51 y 0.09 .

\section{Energía}

Las concentraciones de energía presente en las barras de chocolate son similares, con una media general de 607 Kcal y un Coeficiente de variación de 8.02\% (Cuadro 1). Los valores mayores entre las muestras lo obtuvieron DICYT-H 259 (634 Kcal) y el DICYT-H 267 (628 Kcal). Mientras que los menores valores registrados fueron DICYT-H 264 (558) y el CCN-51 (567). En la Base de Datos de Alimentos (BADALI) y de la Base de Datos Española de Composición de Alimentos (BEDCA), los mayores valores de energía se obtuvieron en las semillas de cacao tras el tostado (>550 Kcal) y chocolate con leche y almendras (549 Kcal), y los menores registrados fueron registrados en Cacao en polvo desgrasado $(255 \mathrm{Kcal})$ y Cacao en polvo azucarado (390 Kcal).

"The Cocoa. Plan Un trabajo conjunto, paso a paso" indica valores para cacao en polvo $(335 \mathrm{Kcal})$ y para chocolate con leche $(546 \mathrm{Kcal})$ similares obtenidos en las barras de chocolate negro (Cirera, 2011). Así, Vera et al. (2014), caracterizaron los atributos físico-químicos de almendras y características sensoriales de pastas de quince clones de cacao; doce de tipo Nacional y tres testigos (CCN-51, EET-103 e IMC-67) e indican que las medias obtenidas no presentan diferencias estadísticas. Williams et al. (2009), señalan un promedio de energía 502 Kcal menor al promedio obtenido en las barras de chocolate negro.

\section{Análisis organoléptico}

En el análisis organoléptico a las barras de chocolate negro elaboradas con siete genotipos experimentales se explica un $78.10 \%$ de la varianza total asociada al impacto que tiene las características de las barras de chocolate negro sobre el análisis organoléptico. Para el dulzor se observa que el DICYT-H 259 y el Testigo CCN-51 obtuvieron el mayor dulzor, debido al edulcorante utilizado en la formulación. Respecto al sabor floral lo obtuvo el DICYT-H 262 acompañado con aroma e intensidad frutal muy ligera. Para sabor a cacao, fue superior para el DICYT-H 263, mientras, el DICYT-H 264 obtuvo la mayor astringencia, no obstante, el mayor amargor lo obtuvo el DICYT-H 267 acompañado con cierta astringencia lo que podría atribuirse a concentraciones de polifenoles y taninos en las muestras. 
Chocolate de siete genotipos experimentales de cacao (Theobroma cacao L.)

Cuadro 2. Análisis organoléptico de barras de chocolate negro de siete cruces experimentales de la Finca Experimental "La Represa"

\begin{tabular}{|c|c|c|c|c|c|c|c|c|c|c|c|c|c|c|}
\hline \multirow{2}{*}{$\begin{array}{c}\text { Código } \\
\text { Tratamientos }\end{array}$} & \multicolumn{3}{|c|}{ Generalidades } & \multicolumn{4}{|c|}{ Sabores Básicos } & \multicolumn{4}{|c|}{ Sabores Específicos } & \multicolumn{3}{|c|}{ Defectos } \\
\hline & Aroma & Sabor & Intensidad & Cacao & Acidez & Amargor & Astringencia & Floral & Frutal & Nuez & $\begin{array}{l}\text { Caramelo } \\
\text { o Melaza }\end{array}$ & Moho & Quemado & Contaminado \\
\hline DICYT-H 258 & 3,30 & 6,10 & 6,70 & 5,60 & 4,30 & 5,50 & 5,20 & 2,00 & 1,20 & 0,20 & 1,50 & 0,10 & 0,80 & 0,00 \\
\hline DICYT-H 259 & 4,00 & 6,20 & 5,00 & 3,70 & 2,40 & $\mathbf{2 , 7 0}$ & 3,00 & 2,80 & 2,10 & 0,60 & 1,80 & 0,20 & 0,30 & 0,00 \\
\hline DICYT-H 262 & 4,50 & 6,20 & 6,60 & 6,10 & 4,10 & 5,60 & 5,50 & 2,50 & 2,40 & 1,30 & 1,20 & 0,10 & 0,50 & 0,10 \\
\hline DICYT-H 263 & 5,60 & 7,10 & 6,80 & 6,20 & 5,10 & 6,30 & 7,10 & 3,00 & 2,30 & 0,40 & 1,10 & 0,30 & 1,30 & 0,30 \\
\hline DICYT-H 264 & 2,90 & 6,40 & 6,60 & 5,90 & 5,00 & 7,00 & 6,60 & 2,50 & 2,80 & 0,60 & 0,40 & 0,20 & 0,60 & 0,00 \\
\hline DICYT-H 267 & 4,30 & 7,00 & 6,40 & 6,80 & 5,90 & 7,00 & 6,80 & 2,70 & 1,70 & 0,30 & 0,50 & 0,20 & 0,60 & 0,10 \\
\hline CCN-51 & 2,50 & 6,00 & 3,90 & 4,10 & 1,00 & 1,30 & 2,30 & 2,20 & 2,50 & 0,80 & 3,30 & 0,00 & 0,20 & 0,00 \\
\hline Promedio & 3,87 & 6,43 & 6,00 & 5,49 & 3,97 & 5,06 & 5,21 & 2,53 & 2,14 & 0,60 & 1,40 & 0,16 & 0,61 & 0,07 \\
\hline L.I. (0.05) & 2,89 & 6,02 & 4,97 & 4,42 & 2,39 & 3,01 & 3,46 & 2,21 & 1,65 & 0,26 & 0,5 & 0,07 & 0,28 & $-0,03$ \\
\hline L.S. $(0.05)$ & 4,85 & 6,84 & 7,03 & 6,55 & 5,55 & 7,1 & 6,96 & 2,85 & 2,64 & 0,94 & 2,3 & 0,25 & 0,95 & 0,17 \\
\hline E.E. & 0,40 & 0,16 & 0,42 & 0,43 & 0,64 & 0,83 & 0,71 & 0,13 & 0,20 & 0,13 & 0,36 & 0,036 & 0,13 & 0,04 \\
\hline
\end{tabular}

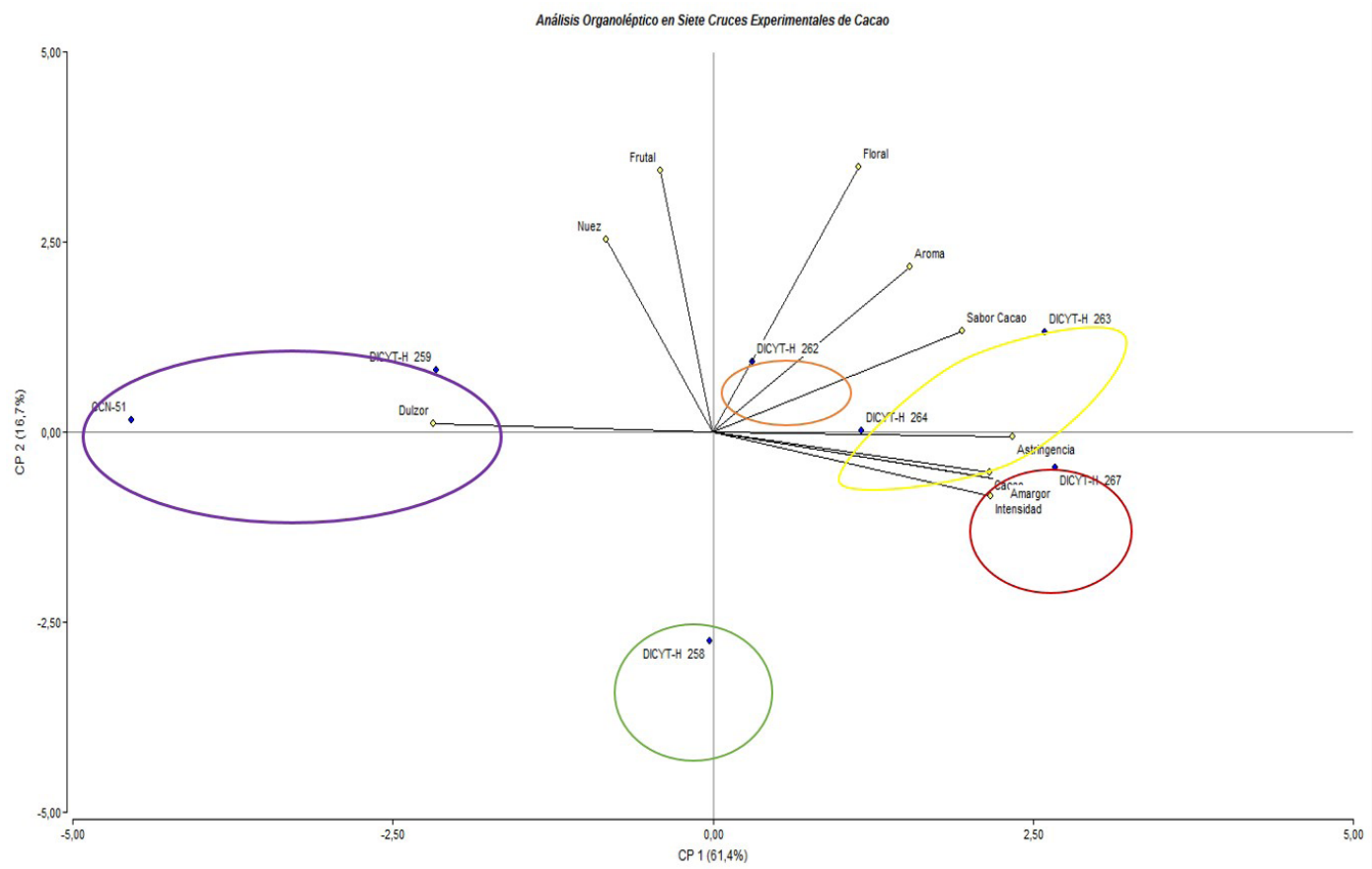

Figura 2. Análisis Organoléptico en Siete Cruces Experimentales de Cacao, FCP-UTEQ

Cuadro 3. Valores registrados de Análisis económico en barras de chocolate negro, FCP-UTEQ

\begin{tabular}{|c|c|c|c|c|c|c|c|}
\hline Rubros & $\begin{array}{l}\text { CCN-51 } \\
\text { (Testigo) }\end{array}$ & $\begin{array}{c}\text { DICYT-H } \\
258\end{array}$ & $\begin{array}{c}\text { DICYT-H } \\
259\end{array}$ & $\begin{array}{c}\text { DICYT-H } \\
262\end{array}$ & $\begin{array}{c}\text { DICYT-H } \\
263\end{array}$ & $\begin{array}{c}\text { DICYT-H } \\
264\end{array}$ & $\begin{array}{c}\text { DICYT-H } \\
267\end{array}$ \\
\hline \multicolumn{8}{|l|}{ Costos variables (USD) } \\
\hline Labores culturales & 1.43 & 2.14 & 2.50 & 2.62 & 3.28 & 2.45 & 2.67 \\
\hline Materia prima & 12.35 & 0.64 & 0.64 & 0.64 & 0.64 & 0.64 & 0.64 \\
\hline Mano de obra directa & 19.74 & 19.74 & 19.74 & 19.74 & 19.74 & 19.74 & 19.74 \\
\hline Costos indirectos (USD) & 13.25 & 13.25 & 13.25 & 13.25 & 13.25 & 13.25 & 13.25 \\
\hline Costos fijos (USD) & 4.42 & 4.43 & 4.43 & 4.43 & 4.43 & 4.43 & 4.43 \\
\hline Total egresos (USD) & 51.20 & 40.20 & 40.56 & 40.69 & 41.34 & 40.51 & 40.74 \\
\hline
\end{tabular}




\section{Análisis económico}

En el cuadro 3 se muestra el análisis económico de la elaboración de barras de chocolate negro exponiendo mayor costo al tratamiento testigo CCN-51 (51.20 USD) y menor costo al tratamiento DICYT-H 258 (40.20 USD). Existe diferencia entre los tratamientos con respecto al costo, pues, al elaborar las barras de chocolante en el CCN-51 se utilizó porcentajes diferentes en la formulación. Sin embargo, las labores culturales (cosecha, poda, fermentado, secado y prevención de enfermedades), las horas de trabajo y costo, varían en cada tratamiento.

\section{Conclusiones}

$\mathrm{E}$ n la evaluación de los parámetros de calidad químicos en la variable porcentaje de grasa, el tratamiento DICYT-H 258 obtuvo mayor porcentaje de grasa $(37.83 \%)$ superando al Testigo $(22.86 \%)$, además de tener menor acidez (2.04\%), la mayor acidez $(8.79 \%)$ y energía (634 Kcal) la obtuvo DICYT-H 259. El tratamiento DICYT-H 262 obtuvo características sabor floral y aroma e intensidad frutal en el análisis organoléptico, acercándolo más al sabor característico del Cacao Fino de Aroma. El tratamiento CCN-51 obtuvo el mayor costo (USD 51.20) en la elaboración de barras de chocolate negro por el uso de más aditivos..

\section{Bibliografía}

Afoakwa, E. [y otros]. 2011. Chemical composition and physical quality characteristics of Ghanaian cocoa beans as affected by pulp pre-conditioning and fermentation [Publicación periódica]. Association of Food Scientists \& Technologists.

Gastón, R. 2016. http://www.iniap.gob.ec/pruebav3/ programa-nacional-de-cacao-del-iniap-desarrollaclones-de-cacao-de-alta-productividad/

Cirera, A. 2011. The Cocoa Plan. Un trabajo conjunto, paso a paso [Publicación periódica]. Nesvida. Marzo de 2011. 12(6).

Fernández, C. 2016. Elaboración de Chocolate: Informe.

Foundation World Cocoa [En línea]. Cision PR Newswire. https://www.prnewswire.com/newsreleases/la-fundacion-mundial-del-cacao-anunciael-establecimiento-de-un-grupo-de-trabajo-decultivadores-de-cacao-en-las-americas-280923342. html.

Furcal, P. 2015. Extracción de nutrientes esenciales en frutos de clones de cacao en producción en dos regiones en Costa Rica [Informe]: Informe de Proyecto/Escuela de Agronomía; Instituto Tecnológico de Costa Rica. pág. 29.

Gómez, M. [y otros]. 2011. Efectos beneficiosos del chocolate en la salud cardiovascular [Publicación periódica]. Nutrición Hospitalaria. - Abril de 2011. 26(2).

Guzmán, J., Gómez, S. 2014. Evaluación sensorial de cacao (Theobroma cacao L.) cultivado en la región del sur del departamento de Bolívar (Colombia) [Publicación periódica]. Revista de Investigación Agraria y Ambiental. - julio-diciembre. 5(2).

Homem, G. [y otros]. 2017. Influencia de factores agroambientales sobre la calidad del clon de cacao (Theobroma cacao L.) pH-16 en la región cacaotera de Bahia, Brasil [Publicación periódica].Ecosistema y Recursos Agropecuarios. Brasil. 4(12).

Lares Mary [y otros]. 2016. Cambios de las propiedades físico-químicas y perfi 1 de ácidos grasos en cacao de Chuao, durante el benefi cio [Publicación periódica]. Scielo. Octubre. 63(1,2).

Ledesma, G. 2017. Evaluación de la eficacia de tres tipos de injertos en cacao Nacional (Theobroma cacao L.), en patrones de tres edades, en la zona de Ventanas, provincia de Los Ríos. [Informe]: Escuela de Ingeniería Agronómica. Universidad Estatal de Bolívar. Guaranda. 75 p.

Maldonado, J. 2016. Perspectivas Económicas y Financieras en el cultivo de cacao CCN51 vs cacao fino de Aroma para la decisión de inversión de la empresa Famisa [Informe]: Facultad de Ciencias Sociales y Humanísticas; Escuela Superior Politécnica del Litoral. Guayaquil. 79 p.

Molina, M. 2017. Investigación para determinar la aceptación de los pobladores del distrito metropolitano de Quito de bombones en base a chocolate Ambateño [Informe]. Quito.

Oliveras, J. 2007. La elaboración del chocolate, una técnica dulce y ecológica [Publicación periódica] Técnica Industrial. - Marzo-Abril. 268.

Proecuador [En línea]. 2017. Proecuador. https://www. proecuador.gob.ec/compradores/oferta-exportable/ cacao-y-elaborados/

Ropero, A. 2016. Cacao y Chocolate [Publicación periódica]. Badali.

Ruíz, M. [y otros]. 2014. Influencia de la época de cosecha en la calidad del licor de cacao tipo Nacional [Publicación periódica]. ESPAMCIENCIA. 5(2).

Salinas, N., Bolivar, W. 2012. Ácidos grasos en chocolates venezolanos y sus análogos [Publicación periódica]. Scielo. Junio. 25(1).

Soto, A., Caballero, L. 2011. Adición de hierro hemo, proveniente de hemoglobina bovina a un chocolate de consumo directo [Publicación periódica]. Bistua:Revista de la Facultad de Ciencias Básicas. Enero. 9(1).

Tigselema, S. 2018. Elaboración de chocolate de siete genotipos experimentales de cacao (Theobroma 
cacao L.) Seleccionados en la Finca Experimental La Represa [Informe]. Quevedo.

Vera, J. [y otros]. 2014. Atributos físicos-químicos y sensoriales de las almendras de quince clones de cacao nacional (Theobroma cacao L.) en el Ecuador [Publicación periódica]. Ciencia y Tecnología. julio-diciembre. 7(2).

Williams, S., Tamburic, S., Lally, C. 2009. Eating chocolate can significantly protect the skin from UV light [Publicación periódica]. Journal of Cosmetic Dermatology. 8(3): 169-173. 